Chapter 4

\title{
Condensins, Chromatin Remodeling and Gene Transcription
}

\author{
Laurence O. W. Wilson and Aude M. Fahrer \\ Additional information is available at the end of the chapter \\ http://dx.doi.org/10.5772/55732
}

\section{Introduction}

Condensin complexes condense chromosomes during mitosis, turning the diffuse interphase chromosomes into the familiar X-shaped compact chromosomes that segregate during cell division. More recently a second role for condensins has emerged, in the epigenetic regulation of interphase gene transcription. This second fascinating role is very difficult to study since defects in condensin will usually interfere with mitosis and result in cell death. While several excellent reviews of condensin function have recently been published (see [1-3]), in this article we concentrate on the epigenetic functions of condensins and how they can be studied. We also provide the first summary of condensin protein splice forms, a largely overlooked contributor to condensin variation.

The DNA within a cell is too large to fit inside if left in its unwound state. In order to accommodate the genetic material, a cell packages this DNA as chromati:, a combination of DNA bound to protein. The DNA is wound around protein complexes known as histones to form nucleosomes, which form a "beads-on-a-string" structure. These nucleosomes can be compacted further to produce a highly condensed structure that fits inside the nucleus of a cell.

The regulation of this structure is vital for cell growth and survival. During mitosis, chromatin must be unwound so that it can be properly replicated and then repackaged into sister chromosomes that must then be segregated into the dividing cells. Defects in any of these processes can result in cell death. Additionally, the compact nature of the chromatin limits the cell's transcription machinery from properly interacting with its targets, preventing gene transcription. In order to counter this, selected regions of chromatin must be unwound during interphase to allow the genes present to be transcribed. The chromatin structure of the cell is therefore under tight control. Changes in chromatin structure are thought to occur via three 
broad mechanisms; 1 . methylation of DNA; 2. covalent modification of histones; 3 . at a "higher level" by tethering distant regions of DNA.

The condensin protein complexes, originally identified in Xenopus egg extracts [4], function through the third pathway: inducing condensation in chromatin structure through the introduction of positive super-coils, and by tethering together distinct regions of DNA $[5,6]$. This ability to condense chromatin has been found to be central to the compaction of chromosomes during mitosis.

Vertebrates possess two condensins, known as condensin I and condensin II, each consisting of 5 proteins. A dimer of Structural Maintenance of Chromosome (SMC) proteins 2 and 4 forms the core of both condensin I and II. SMC proteins are large proteins (1000 - 1300 amino acid long) which fold so that their amino and carboxy termini join to form an ATPase [7]. SMC2 and 4 form a dimer with a V-shape, with the ATPase domains of each protein localized to the tips of the V. This dimer of SMC proteins then associates with either Ncaph, Ncapg and Ncapd2 to form condensin I or with Ncaph2, Ncapg2 and Ncapd3 to form condensin II (Figure 1) [8, 9].

\section{Condensin I}

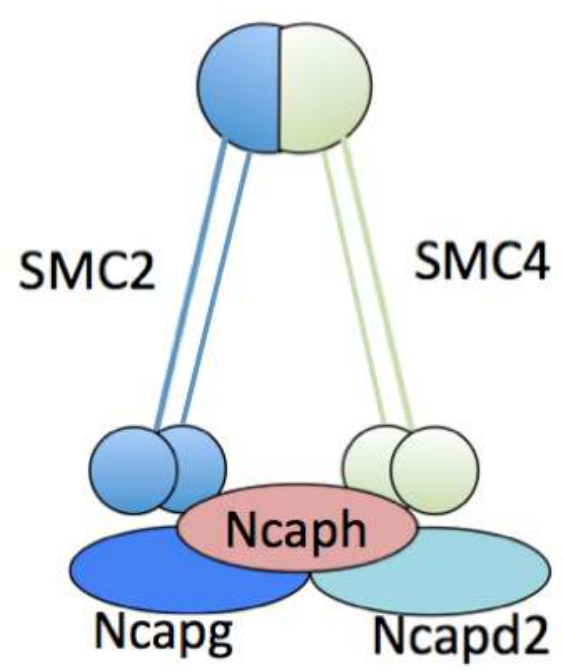

\section{Condensin II}

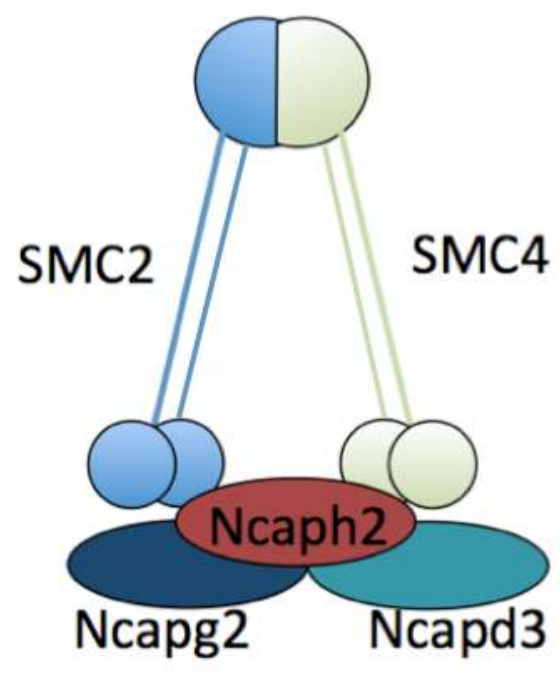

Figure 1. Schematic representation of the condensin complexes. A heterodimer of SMC2 and SMC4 combines with either Ncaph, Ncapg and Ncapd2 to form condensin I or with Ncaph2, Ncapg2 and Ncapd3 to form condensin II. 
Condensins were initially studied in the context of mitotic chromosomes. Mutations interfering with the function of either condensin complex results in aberrant chromosome formation, and a complete loss of function of either complex is lethal $[9,10]$. The emerging evidence for chromatin structure's role in gene transcription however has lead to idea that condensins may play roles beyond mitosis, acting in interphase to influence gene transcription through the reorganization of chromatin [11-13]. Subsequently, evidence has begun to emerge which shows that condensins are much more than architects of chromatin structure, they are also important regulators of gene transcription.

The vital role condensins play in mitosis makes their study difficult; disruption of their function through means such as mutations or transcriptional interference often fatally disrupts cell division. A variety of methods of study have therefore been employed in order to gain insight into the complexes' roles in mitosis and gene regulation.

\section{The study of condensin homologs}

An alternative to studying a protein directly is to study functionally and structurally similar homologs. Such homologs may provide insight into a protein of interest through shared mechanisms, but prove easier to study. Such an approach has been used to study condenins and provided great insight into how they might regulate gene transcription.

One of the first examples of condenins regulating gene expression was the discovery of a condensin homolog in C. elegans known as the Dosage Compensation Complex (DCC) [14]. In most species, the sexes are determined by a difference in the presence of specific chromosomes (such as the $\mathrm{X}$ and $\mathrm{Y}$ chromosomes in human). In C. elegans the population is divided into males, which possess a single $X$ chromosome, and hermaphrodites, which possess two copies of the $X$ chromosome. The increased number of $X$ chromosome present in hermaphrodites would lead to a two-fold increase in X-linked gene expression with potentially dire consequences unless properly regulated [15]. To counter this, hermaphrodite $C$. elegans can reduce the expression of their X-linked genes by half, resulting in normal levels of transcription [15].

The DCC is comprised of SMC proteins DPY-27 and MIX-1 as well as DPY-26, DPY-28 and Capg1 [16-20]. The DCC then associates with a second 5-protein complex consisting of SDC-1, SDC-2, SDC-3, DPY-21 and DPY-30. The structure of the DCC differs from the worm condensin I complex only in the presence of DPY-27, which is replaced by SMC-4 in condensin I, yet despite the similarities has a different function.

Studies have shown that the DCC is directed towards the $\mathrm{X}$ chromosome by a combination of histone modifications and specific DNA motifs. The histone variant H2A.Z (called HTZ-1 in C. elegans) is found on the X chromosome of C. elegans. Disrupting the expression of this histone using RNAi's was shown to disrupt DCC binding. The disruption was not caused by a decrease in DCC protein levels, which suggests the histone assists in the binding/recruitment of the complex to the $X$ chromosome [21, 22]. Once recruited to the 
correct chromosome, the complex binds to the DNA through the recognition of specific sequence motifs in what is now known to be a two-step process. First, the DCC recognizes and binds to recruitment elements on X (or rex) sites. Once bound, the DCC is then distributed along the $X$ chromosome by migrating to dependent-on- $X$ (or dox) sites. The result is the distribution of the DCC along the full length of the $X$ chromosome, allowing it to exert its effects over the entire chromosome [23].

A second, less widely known example of a condensin- related protein was identified in a mouse study looking for epigenetic regulators. Blewitt et al. identified the gene SMC Hinge domain containing 1 (SMCHD1) [24]. While not a full homolog of SMC, it does bear similarities and further investigation showed that it plays a crucial role in $\mathrm{X}$-inactivation in mice, similar to the DCC in C. elegans [25]. Further study of this protein may provide insights into the function of SMC complexes such as condensin.

\section{The study of condensins through knock-outs}

Removal of a gene of interest, and study of the resulting phenotype, is a common method for studying the function of a gene. The vital role condensins play in mitosis prevents such an approach, as knock-outs are often lethal. Knockouts in SMC2, SMC4, Ncapd2, Ncapg and Ncaph have all proven to be lethal [26-34]. The majority of the knock-out work has been conducted in yeast, bacteria, Drosophila, and C. elegans. A study in 2004 generated the only reported mammalian phenotype for a condensin knockout: a mouse strain with a deletion of the condensin II Ncapg2 protein [35]. The resulting knock-outs were embryonic lethal, with the mice fetuses dying almost immediately after implantation due to a catastrophic disruption in cell growth and division.

An alternative to the use of full knock-outs is the generation of conditional knock-outs. Cell lines can be engineered to turn-off expression of target genes when exposed to specific signals (such as the addition of doxycylcin). Expression of the gene is then lost over time and a resulting phenotype emerges. Chicken cell lines have now been created that stop expression of select condensin genes upon exposure to doxycycline [36]. Whereas a normal knock-out of these genes would likely prove lethal and prevent any sort of study, the gradual emergence of the phenotype in these conditional knock-outs can be studied. Such an approach has been used to investigate the different contributions of condensin I and condensin II to mitotic-chromosome formation, showing differences in phenotype between knock-outs of the two complexes. The results suggest that condensin II is required for the formation of the chromosome scaffold and for providing rigidity, while condensin I acts to compact the chromatin around the scaffold. While only used so far to study the role of these complexes in mitosis, their use could be expanded to investigate the role condensins play during interphase. Gene expression profiling of these cell lines could be carried out to look for evidence of the direct regulation of transcription by condensins. 


\section{Knock-down and overexpression studies}

Altering the expression of target proteins, either through over- or under-expression can provide an alternative to knock-out studies, producing informative phenotypes while avoiding the lethal side-effects often associated with complete removal of a protein. Such approaches have been used in Drosophila to great effect, providing insights into the functions of Condensins.

The Drosophila Barren protein, a homolog of Ncaph and component of the Drosophila Condensin I complex, was investigated using a knock-down approach [37]. The study showed that fly Ncaph interacts directly with the Polycomb group protein polyhomeotic. Polycomb group proteins are a group of proteins responsible for repressing transcription in various regions across the genome and for maintaining this silenced state through the life of the fly. This is achieved by the binding of these proteins to specific elements within the genome and the compaction of neighboringDNA.Thephysicalinteraction withNcaphsuggeststhat thiscompactionisachieved through the recruitment of Condensin via the polycomb group proteins to the target region of the genome. The authors demonstrated a specificinstance of this, showing the fly requires Ncaph to silence the abdominal- $B$ gene by inducing its binding to an upstream silencer. Reducing the levels of Ncaph through knock-downs greatly diminished the silencing of this gene, highlighting the role of condensin I in transcriptional regulation.

A similar approach was used in a second Drosophila study [38]. The authors noted that Drosophila larvae with mutations in retinoblastoma family protein RBF1 often showed defects in chromsome condensation during mitosis. Further investigation showed a direct interaction between RBF1 and the Drosophila Ncapd3 protein. The authors showed that this interaction was required for proper loading of condensin II onto the chromosome. A reduction in the levels of RBF1 greatly diminished the capacity of condensin II to bind to the chromatin, but could be compensated by over-expression of Ncapd3.

Over-expression was also used to explore the role of murine Ncapg2/MTB in erythropoiesis [39]. Ncapg2 was found to be upregulated in erythroid cell lines. Further investigation found the protein was capable of inhibiting SCL/E12 mediated transcription. Over-expression of the protein was also shown to be sufficient to induce terminal differentiation of erythroleukemia cell lines. While the precise mechanism of Ncapg2-mediated differentiation is still poorly understood, the authors proposed two non-exclusive hypotheses. During erythroid cell development, the chromatin becomes progressively more compacted as the cell matures and the nucleus is ultimately ejected [40]. An increased level of Ncapg2 could result in a higher level of condensin II, in turn leading to a higher level of chromatin compaction. Alternatively, the authors proposed that Ncapg2 may act independently of the Condensin complex, cooperating with additional enzymes to induce red blood cell development.

\section{Mutation studies}

In 2007, our laboratory reported the first viable mammalian mutant of a condensin protein; the Nessy mouse, with a point mutation in the Ncaph2 (kleisin $\beta$ ) gene [41]. The mutant was 
identified as part of an ENU-mutagenesis screen for immunological phenotypes, and has a partial block in T-cell development. The mutation was mapped to a region in mouse chromosome 15 and ultimately identified as a T to A substitution in exon 1 of the Ncaph2 gene. The mice are viable and appear otherwise normal. The phenotype therefore is not due to impairment of cell division. Indeed, evidence was obtained that at one stage of T-cell development (the $\mathrm{CD} 4^{+} \mathrm{CD} 8^{+}$double positive stage) Nessy thymocytes undergo more cell divisions than their wild-type counterparts. No defects were identified in the B-cell lineage. These studies provide the first evidence for a cell-type specific role of a condensin complex component. A subsequent study pinpointed functional deficiencies in mature Nessy T-cells [42]. More recently a study by Rawlings et al. presented evidence suggesting that the point mutation in the Nessy mouse prevents Condensin II from condensing the chromatin at a key stage during thymocyte development, resulting in aberrant gene transcription [43].

Another ENU mutagenesis screen, this time conducted on zebrafish, identified a mutation in condensin I subunit Ncapg which caused a reduction in the number of retinal cells [44]. In addition, the animals displayed a high incidence of polyploidy suggesting defects in cell division.

\section{Study of alternative splice forms}

Until recently, it has been assumed that the genes encoding the components of the condensin complexes each produce only a single protein. Recently however, it has emerged that this may not be the case. In the same study that described the mutant mouse model, our laboratory also showed that mouse Ncaph2 can undergo alternative splicing to produce multiple distinct protein isoforms [41]. We have since studied this in more detail, showing that the mouse Ncaph2 gene is capable of encoding at least 6 unique protein isoforms [45]. The first exon of the gene can be translated into one of three forms. In addition, we detailed the inclusion of an additional alanine residue between exons 15 and 16 resulting from NAGNAG variation. Our data show that the amino-termini variants are ubiquitous throughout the mouse, expressed at similar levels in all tissues tested.

The remaining components of the condensin complexes have never been studied to see if they undergo alternative splicing despite annotated instances existing in the NCBI and ENSEMBL database. We recently completed a large scale study into alternative splicing that identified additional instances of splice variation in Ncaph2 and Ncapd2. A comprehensive summary of the splice variants of all condensin subunits is presented in Table 1. None of the splice variants have been characterized yet, so it remains to be seen if they are functionally unique. Their existence however, raises the possibility that these distinct isoforms may regulate the different functions of condensins. Inclusion of one splice form over another may switch the complex from an architect of chromosomes to a regulator of gene function, providing an elegant method to regulate function. 


\begin{tabular}{|c|c|c|c|}
\hline \multicolumn{4}{|l|}{ Mouse } \\
\hline Protein & Splice Type & Description of splicing & Reference \\
\hline SMC2 & $\begin{array}{l}\text { Partial } \\
\text { transcription }\end{array}$ & $\begin{array}{l}\text { Transcription of only the first } 11 \text { exons, resulting in a truncated } \\
\text { protein }\end{array}$ & ENSEMBL \\
\hline \multirow[t]{2}{*}{ SMC4 } & $\begin{array}{l}\text { Partial } \\
\text { transcription }\end{array}$ & Transcription of only the first 8 exons, resulting in a truncated protein & ENSEMBL \\
\hline & $\begin{array}{l}\text { Alternate splice } \\
\text { site }\end{array}$ & $\begin{array}{l}\text { Alternate in frame splice site at the start of exon 3, resulting in the } \\
\text { deletion of } 25 \text { amino-acids from the beginning of the exon }\end{array}$ & ENSEMBL \\
\hline Ncapd2 & Cryptic exon & $\begin{array}{l}\text { Inclusion of a cryptic exon between exons } 20 \text { and } 21 \text { and initiating } \\
\text { translation at an alternate start codon produces a truncated protein } \\
\text { with a unique amino-terminus }\end{array}$ & $\begin{array}{l}\text { Wilson et al } \\
\text { (submitted) }\end{array}$ \\
\hline Ncapd3 & Exon skipping & $\begin{array}{l}\text { Skipping of exons } 25-28 \text {, resulting in the deletion of } 283 \text { amino- } \\
\text { acids }\end{array}$ & ENSEMBL \\
\hline \multirow[t]{3}{*}{ Ncaph2 } & $\begin{array}{l}\text { Alternate splicing } \\
\text { of exon } 1\end{array}$ & $\begin{array}{l}\text { The protein can splice the first coding exon in one of three ways, } \\
\text { producing proteins with distinct amino-termini }\end{array}$ & $\begin{array}{l}\text { Theodoratos et al, } \\
2012\end{array}$ \\
\hline & Cryptic exon & $\begin{array}{l}\text { Inclusion of a cryptic exon between exons } 5 \text { and } 6 \text {, as well as initiating } \\
\text { translation from an alternate start site produces a protein with a } \\
\text { unique amino-terminus }\end{array}$ & $\begin{array}{l}\text { Wilson et al } \\
\text { (submitted) }\end{array}$ \\
\hline & $\begin{array}{l}\text { NAG-NAG } \\
\text { variation }\end{array}$ & $\begin{array}{l}\text { Potential choice of a second splice site can introduce an extra residue } \\
\text { between exons } 15 \text { and } 16\end{array}$ & $\begin{array}{l}\text { Theodoratos et al, } \\
2012\end{array}$ \\
\hline \multicolumn{4}{|l|}{ Human } \\
\hline Protein & Splice Type & Description of splicing & Reference \\
\hline SMC2 & Cryptic exon & $\begin{array}{l}\text { Inclusion of a cryptic exon after exon } 23 \text { introduces an alternate stop } \\
\text { codon }\end{array}$ & ENSEMBL \\
\hline \multirow[t]{2}{*}{ SMC4 } & $\begin{array}{l}\text { Alternate splice } \\
\text { site }\end{array}$ & $\begin{array}{l}\text { Alternate in frame splice site at the start of exon 3, resulting in the } \\
\text { deletion of } 25 \text { amino-acids }\end{array}$ & ENSEMBL \\
\hline & Exon skipping & Skipping of exon 19 , resulting in the deletion of 58 amino-acids & ENSEMBL \\
\hline \multirow[t]{2}{*}{ Ncaph } & Exon skipping & Skipping of exon 2, resulting in the deletion of 136 amino-acids & ENSEMBL \\
\hline & $\begin{array}{l}\text { Alternate splice } \\
\text { site }\end{array}$ & $\begin{array}{l}\text { Alternate in frame splice site at the start of exon 2, resulting in the } \\
\text { deletion of } 11 \text { amino-acids }\end{array}$ & ENSEMBL \\
\hline \multirow[t]{2}{*}{ Ncapd2 } & $\begin{array}{l}\text { Alternate splice } \\
\text { site of exon } 1\end{array}$ & $\begin{array}{l}\text { Alternate splice site at the end of exon } 1 \text { and initiating translation at } \\
\text { an alternate start codon }\end{array}$ & $\begin{array}{l}\text { Wilson et al } \\
\text { (submitted) }\end{array}$ \\
\hline & Exon skipping & Skipping of exon 4 , resulting in the deletion of 45 amino-acids & ENSEMBL \\
\hline \multirow[t]{2}{*}{ Ncapd3 } & Alternate exon & Can be transcribed with one of two first exons & ENSEMBL \\
\hline & Cryptic exon & $\begin{array}{l}\text { Inclusion of a cryptic exon after exon 12, creating an alternate stop- } \\
\text { codon }\end{array}$ & ENSEMBL \\
\hline \multirow[t]{2}{*}{ Ncaph2 } & $\begin{array}{l}\text { Alternate splice } \\
\text { site of exon } 9\end{array}$ & $\begin{array}{l}\text { Alternate splice site of exon } 9 \text { creating an alternate stop-codon, } \\
\text { resulting in a truncated protein with an alternate carboxy-terminus }\end{array}$ & $\mathrm{NCBI}$ \\
\hline & $\begin{array}{l}\text { NAG-NAG } \\
\text { variation }\end{array}$ & $\begin{array}{l}\text { Potential choice of a second splice site can introduce an extra residue } \\
\text { between exons } 15 \text { and } 16\end{array}$ & $\begin{array}{l}\text { Theodoratos et al, } \\
2012\end{array}$ \\
\hline
\end{tabular}

Table 1. Splice variations of the condensin components 


\section{Separation of function}

The separation of the condensin complexes' functions in mitosis and gene regulation remains an inherently difficult process. The Nessy mouse represents the first true separation of function in a vertebrate model, the point mutation disrupting the cell-specific role of condensin II while leaving its mitotic role unperturbed. A previous study in bacteria reported a similar separation. Mutations in the kleisin protein ScpA in Bacillius subtillis were found to disrupt the DNA repair pathways as well as resulting in the deregulation of specific genes while leaving the mitotic function intact [46].

Such results show that the functions of condensin can be separated, but is still unknown how this occurs. The alternative splice forms may play a role in the regulation of condensin function. Inclusion of alternate isoforms may influence weather the complex acts as a compacter of chromosomes or as a regulator of gene transcription. In our original 2007 paper, we showed that the Nessy mutation could be rescued by reintroduction of only one splice variant (designated as the Long-form). The rescue was of the peripheral phenotype (CD44 ${ }^{\text {hi }} \mathrm{T}$-cells in the spleen). Technical limitations meant that rescue of the thymus phenotype could not be confirmed. Rescue with different splice forms, may have provided additional insight.

Condensins have also been shown to interact with additional proteins capable of regulating their function. Once such protein is protein phosphatase 2A (PP2A), which is capable of dephosphorylating the Ncapg subunit of condensin I, inhibiting its function [47]. This activity has been shown to be important for gene-bookmarking. During cell division, individual cells must "remember" their lineage. Promoters of select genes remain un-compacted during the process of division, allowing for rapid expression of the genes after mitosis [48, 49]. PP2A was found to be important for leaving these genes un-compacted. PP2A interacts with the TATAbinding protein (TBP) which binds to the promoter regions of active genes during mitosis. The combination of the two proteins then dephosphorylate nearby condensin, preventing condensation of chromatin and allowing the genes to become active shortly after division [50].

Additionally, condensin II has been shown to bind to specific histone markers. Ncapg2 and Ncapd3 were found to recognize mono-methylation of H4K20, inducing condensin II to bind to the DNA whereas di-methylation of the histone was found to lead to dissociation of the complex [51]. Based on this finding, the authors suggested a model for chromosome condensation during mitosis; once the cell progresses into mitosis, a demethlyase converts H4K20me2 to H4K20me1, inducing condensin II to bind and compact the chromatin into chromosomes. Upon proper separation of the chromosomes, H4K20me1 is converted back to H4K20me2 causing condensin II to separate from the chromatin, resulting in the DNA returning to an open state. This mechanism also provides a possible explanation for how condensins could be targeted to distinct locations along the genome during interphase. Modification of histones at specific loci could recruit the condensin II complex, inducing compaction of the surrounding chromatin and repression of transcription.

Most studies have assumed that the function of condensins in mitosis and gene regulation is similar: the compaction of chromatin through the tethering of distinct regions. However, this 
has never been experimentally demonstrated. In fact, despite its similarities to the condensin complex, the C. elegans DCC is thought to regulate gene expression through the modification of histones [52], and/or through the recruitment of secondary proteins to the $\mathrm{X}$ chromosome [53] rather than by physically restructuring chromatin. Thus, while condensins may exert their epigenetic/ transcriptional regulation function by compacting DNA, it is also entirely possible that they use a different mechanism. Separation of function mutants, such as the condensin II Nessy mutant mouse, are likely to be the key to answering this fundamental question.

\section{Concluding remarks}

It has become clear that condensins have a role in epigenetic/transcriptional regulation in addition to their better-studied role in chromosome compaction during mitosis. The vital role of condensins makes the study of these complexes difficult, with presently only 3 vertebrate models studied, one of which is embryonic lethal. However, numerous studies using a variety of methods have managed to glean insights into the function of these complexes. Despite the progress of the last decade, there is still much left to be discovered about the condensins and especially how they regulate gene transcription.

\section{Author details}

Laurence O. W. Wilson and Aude M. Fahrer

Research School of Biology, College of Medicine, Biology and Environment, The Australian National University, Canberra, Australia

\section{References}

[1] Hirano T: Condensins: universal organizers of chromosomes with diverse functions Genes Dev (2012).

[2] Hudson, D. F, \& Marshall, K. M. Earnshaw WC: Condensin: Architect of mitotic chromosomes. Chromosome Res (2009).

[3] Wood, A. J, \& Severson, A. F. Meyer BJ: Condensin and cohesin complexity: the expanding repertoire of functions. Nat Rev Genet (2010).

[4] Hirano, T, \& Mitchison, T. J. A heterodimeric coiled-coil protein required for mitotic chromosome condensation in vitro. Cell (1994).

[5] Bazett-jones, D. P, \& Kimura, K. Hirano T: Efficient supercoiling of DNA by a single condensin complex as revealed by electron spectroscopic imaging. Mol Cell (2002). 
[6] Kimura, K. Hirano T: ATP-dependent positive supercoiling of DNA by $13 \mathrm{~S}$ condensin: a biochemical implication for chromosome condensation. Cell (1997).

[7] Hirano T: The ABCs of SMC proteins: two-armed ATPases for chromosome condensationcohesion, and repair. Genes Dev (2002).

[8] Hirano, T, \& Kobayashi, R. Hirano M: Condensins, chromosome condensation protein complexes containing XCAP-C, XCAP-E and a Xenopus homolog of the Drosophila Barren protein. Cell (1997).

[9] Ono, T, Losada, A, Hirano, M, Myers, M. P, \& Neuwald, A. F. Hirano T: Differential contributions of condensin I and condensin II to mitotic chromosome architecture in vertebrate cells. Cell (2003).

[10] Ono, T, Fang, Y, \& Spector, D. L. Hirano T: Spatial and temporal regulation of Condensins I and II in mitotic chromosome assembly in human cells. Mol Biol Cell (2004).

[11] Legagneux, V, \& Cubizolles, F. Watrin E: Multiple roles of Condensins: a complex story. Biol Cell (2004).

[12] Cobbe, N, \& Savvidou, E. Heck MM: Diverse mitotic and interphase functions of condensins in Drosophila. Genetics (2006).

[13] Hagstrom, K. A. Meyer BJ: Condensin and cohesin: more than chromosome compactor and glue. Nat Rev Genet (2003).

[14] Chuang, P. T, \& Lieb, J. D. Meyer BJ: Sex-specific assembly of a dosage compensation complex on the nematode X chromosome. Science (1996).

[15] Meyer, B. J. Casson LP: Caenorhabditis elegans compensates for the difference in X chromosome dosage between the sexes by regulating transcript levels. Cell (1986).

[16] Chuang, P. T, \& Albertson, D. G. Meyer BJ: DPY-27:a chromosome condensation protein homolog that regulates $C$. elegans dosage compensation through association with the X chromosome. Cell (1994).

[17] Hsu, D. R. Meyer BJ: The dpy-30 gene encodes an essential component of the Caenorhabditis elegans dosage compensation machinery. Genetics (1994).

[18] Lieb, J. D, Capowski, E. E, \& Meneely, P. Meyer BJ: DPY-26, a link between dosage compensation and meiotic chromosome segregation in the nematode. Science (1996).

[19] Lieb, J. D, Albrecht, M. R, \& Chuang, P. T. Meyer BJ: MIX-1: an essential component of the $\mathrm{C}$. elegans mitotic machinery executes $\mathrm{X}$ chromosome dosage compensation. Cell (1998).

[20] Csankovszki, G, Collette, K, Spahl, K, Carey, J, Snyder, M, Petty, E, Patel, U, Tabuchi, T, Liu, H, Mcleod, I, et al. Three distinct condensin complexes control C. elegans chromosome dynamics. Curr Biol (2009). 
[21] Petty, E. L, Collette, K. S, Cohen, A. J, \& Snyder, M. J. Csankovszki G: Restricting dosage compensation complex binding to the $X$ chromosomes by H2A.Z/HTZ-1. PLoS Genet (2009). e1000699.

[22] Petty, E, \& Laughlin, E. Csankovszki G: Regulation of DCC localization by HTZ-1/ H2A.Z and DPY-30 does not correlate with H3K4 methylation levels. PLoS One (2011). e25973.

[23] Mcdonel, P, Jans, J, \& Peterson, B. K. Meyer BJ: Clustered DNA motifs mark X chromosomes for repression by a dosage compensation complex. Nature (2006).

[24] Blewitt, M. E, Vickaryous, N. K, Hemley, S. J, Ashe, A, Bruxner, T. J, Preis, J. I, \& Arkell, R. Whitelaw E: An N-ethyl-N-nitrosourea screen for genes involved in variegation in the mouse. Proc Natl Acad Sci U S A (2005).

[25] Blewitt, M. E, Gendrel, A. V, Pang, Z, Sparrow, D. B, Whitelaw, N, Craig, J. M, Apedaile, A, Hilton, D. J, Dunwoodie, S. L, Brockdorff, N, et al. SmcHD1, containing a structural-maintenance-of-chromosomes hinge domain, has a critical role in $\mathrm{X}$ inactivation. Nat Genet (2008).

[26] Freeman, L, \& Aragon-alcaide, L. Strunnikov A: The condensin complex governs chromosome condensation and mitotic transmission of rDNA. J Cell Biol (2000).

[27] Lavoie, B. D, \& Hogan, E. Koshland D: In vivo dissection of the chromosome condensation machinery: reversibility of condensation distinguishes contributions of condensin and cohesin. J Cell Biol (2002).

[28] Lavoie, B. D, Tuffo, K. M, Oh, S, \& Koshland, D. Holm C: Mitotic chromosome condensation requires Brn1p, the yeast homologue of Barren. Mol Biol Cell (2000).

[29] Steffensen, S, Coelho, P. A, Cobbe, N, Vass, S, Costa, M, Hassan, B, Prokopenko, S. N, Bellen, H, Heck, M. M, \& Sunkel, C. E. A role for Drosophila SMC4 in the resolution of sister chromatids in mitosis. Curr Biol (2001).

[30] Britton, R. A, \& Lin, D. C. Grossman AD: Characterization of a prokaryotic SMC protein involved in chromosome partitioning. Genes Dev (1998).

[31] Biggins, S, Bhalla, N, Chang, A, \& Smith, D. L. Murray AW: Genes involved in sister chromatid separation and segregation in the budding yeast Saccharomyces cerevisiae. Genetics (2001).

[32] Strunnikov, A. V, \& Hogan, E. Koshland D: SMC2, a Saccharomyces cerevisiae gene essential for chromosome segregation and condensation, defines a subgroup within the SMC family. Genes Dev (1995).

[33] Strunnikov, A. V, \& Larionov, V. L. Koshland D: SMC1: an essential yeast gene encoding a putative head-rod-tail protein is required for nuclear division and defines a new ubiquitous protein family. J Cell Biol (1993). 
[34] Saka, Y, Sutani, T, Yamashita, Y, Saitoh, S, Takeuchi, M, \& Nakaseko, Y. Yanagida M: Fission yeast cut3 and cut14, members of a ubiquitous protein family, are required for chromosome condensation and segregation in mitosis. EMBO J (1994).

[35] Smith, E. D, Xu, Y, Tomson, B. N, Leung, C. G, Fujiwara, Y, \& Orkin, S. H. Crispino JD: More than blood, a novel gene required for mammalian postimplantation development. Mol Cell Biol (2004).

[36] Green, L. C, Kalitsis, P, Chang, T. M, Cipetic, M, Kim, J. H, Marshall, O, Turnbull, L, Whitchurch, C. B, Vagnarelli, P, Samejima, K, et al. Contrasting roles of condensin I and condensin II in mitotic chromosome formation. J Cell Sci (2012).

[37] Lupo, R, Breiling, A, \& Bianchi, M. E. Orlando V: Drosophila chromosome condensation proteins Topoisomerase II and Barren colocalize with Polycomb and maintain Fab-7 PRE silencing. Mol Cell (2001).

[38] Longworth, M. S, Herr, A, \& Ji, J. Y. Dyson NJ: RBF1 promotes chromatin condensation through a conserved interaction with the Condensin II protein dCAP-D3. Genes Dev (2008).

[39] Xu, Y, Leung, C. G, Lee, D. C, \& Kennedy, B. K. Crispino JD: MTB, the murine homo$\log$ of condensin II subunit CAP-G2, represses transcription and promotes erythroid cell differentiation. Leukemia (2006).

[40] Rothmann, C, \& Cohen, A. M. Malik Z: Chromatin condensation in erythropoiesis resolved by multipixel spectral imaging: differentiation versus apoptosis. J Histochem Cytochem (1997).

[41] Gosling, K. M, Makaroff, L. E, Theodoratos, A, Kim, Y. H, Whittle, B, Rui, L, Wu, H, Hong, N. A, Kennedy, G. C, Fritz, J. A, et al. A mutation in a chromosome condensin II subunit, kleisin beta, specifically disrupts T cell development. Proc Natl Acad Sci U $S A$ (2007).

[42] Gosling, K. M, Goodnow, C. C, \& Verma, N. K. Fahrer AM: Defective T-cell function leading to reduced antibody production in a kleisin-beta mutant mouse. Immunology (2008).

[43] Rawlings, J. S, Gatzka, M, \& Thomas, P. G. Ihle JN: Chromatin condensation via the condensin II complex is required for peripheral T-cell quiescence. EMBO J (2011).

[44] Seipold, S, Priller, F. C, Goldsmith, P, Harris, W. A, \& Baier, H. Abdelilah-Seyfried S: Non-SMC condensin I complex proteins control chromosome segregation and survival of proliferating cells in the zebrafish neural retina. BMC Dev Biol (2009).

[45] Theodoratos, A, Wilson, L. O, \& Gosling, K. M. Fahrer AM: Splice variants of the condensin II gene Ncaph2 include alternative reading frame translations of exon 1. FEBS J (2012). 
[46] Dervyn, E, Noirot-gros, M. F, Mervelet, P, Mcgovern, S, Ehrlich, S. D, \& Polard, P. Noirot P: The bacterial condensin/cohesin-like protein complex acts in DNA repair and regulation of gene expression. Mol Microbiol (2004).

[47] Yeong, F. M, Hombauer, H, Wendt, K. S, Hirota, T, Mudrak, I, Mechtler, K, Loregger, T, Marchler-bauer, A, Tanaka, K, \& Peters, J. M. Ogris E: Identification of a subunit of a novel Kleisin-beta/SMC complex as a potential substrate of protein phosphatase 2A. Curr Biol (2003).

[48] Larsen, A. Weintraub H: An altered DNA conformation detected by S1 nuclease occurs at specific regions in active chick globin chromatin. Cell (1982).

[49] Michelotti, E. F, \& Sanford, S. Levens D: Marking of active genes on mitotic chromosomes. Nature (1997).

[50] Xing, H, \& Vanderford, N. L. Sarge KD: The TBP-mitotic complex bookmarks genes by preventing condensin action. Nat Cell Biol (2008). , 2A.

[51] Liu, W, Tanasa, B, Tyurina, O. V, Zhou, T. Y, Gassmann, R, Liu, W. T, Ohgi, K. A, Benner, C, Garcia-bassets, I, Aggarwal, A. K, et al. PHF8 mediates histone H4 lysine 20 demethylation events involved in cell cycle progression. Nature (2010).

[52] Wells, M. B, Snyder, M. J, \& Custer, L. M. Csankovszki G: Caenorhabditis elegans dosage compensation regulates histone $\mathrm{H} 4$ chromatin state on $\mathrm{X}$ chromosomes. $\mathrm{Mol}$ Cell Biol (2012).

[53] Jans, J, Gladden, J. M, Ralston, E. J, Pickle, C. S, Michel, A. H, Pferdehirt, R. R, Eisen, M. B, \& Meyer, B. J. A condensin-like dosage compensation complex acts at a distance to control expression throughout the genome. Genes Dev (2009). 
